\title{
The Monodromic Singularity to Piecewise Linear Vector Fields
}

\author{
Claudio A. Buzzi, Claudio G. Pessoa, \\ Depto de Matemática, IBILCE, UNESP, \\ 15054-000, São José do Rio Preto, SP \\ E-mail: buzzi@ibilce.unesp.br, pessoa@ibilce.unesp.br,
}

João C. R. Medrado

UFG - Instituto de Matemática e Estatística

Campus Samambaia

74001-970, Goiânia, GO

E-mail: medrado@ufg.br.

\begin{abstract}
Consider in $\mathbb{R}^{2}$ the semi-planes $N=\{y>0\}$ and $S=\{y<0\}$ having as common boundary the straight line $D=\{y=0\}$. In $N$ and $S$ are defined linear vector fields $X$ and $Y$, respectively, leading to a discontinuous polynomial vector field $Z=(X, Y)$. If the vector fields $X$ and $Y$ satisfy suitable conditions, they produce a transition flow from a segment of the splitting line to another segment and this produces a generalized singular point on the line. This point can be a focus or a center. In this paper we give necessary and sufficient conditions to a D-singular point be a monodromic.
\end{abstract}

Keywords: Monodromic singularity, discontinuous linear vector fields.

\section{Introduction}

One of the most accomplished stability theories for dynamical systems is that of AndronovPontryagin [2] and Peixoto [9] for $C^{1}$ vector fields in the plane and on surfaces. Elements of this theory provide characterization and genericity results for structurally stable vector fields. Extensions of this theory to the class of discontinuous, piecewise smooth, vector fields have been provided by Filippov [4] and Kozlova [7]. The need for such an extended theory goes back to Andronov et al. [1].

In [4], Filippov defined the rules (revisited below) for the transition of the orbits crossing the line $D$ of discontinuity which separates two regions $N$ and $S$ on which the field is smooth, given by $X$ and $Y$ respectively. He also prescribed when the orbit slides along $D$. This leads to an orbit structure that is not always a flow on the surface obtained glueing $N$ and $S$ along $D$. The work of Kozlova [7, 4] pursues the setting established by Filippov.

In [12], Sotomayor and Teixeira developed the regularization method, taking as domain the sphere $S^{2}$. This method consists in defining a one parameter family of continuous vector fields that approaches the discontinuous one, when the parameter goes to zero. To this end, a transition function $\varphi$ is used to average $X$ and $Y$ in order to get the family of continuous vector fields. Sotomayor and Teixeira provided conditions on $Z=(X, Y)$, which imply that the regularized vector fields are structurally stable for small values of the parameter. Moreover, Sotomayor and Machado [8] applied the method outlined above to the case of a planar region $M$, with a smooth $\partial M$. The conditions given in [12] are adapted to this case and the genericity, not discussed in [12], is established explicitly there. 


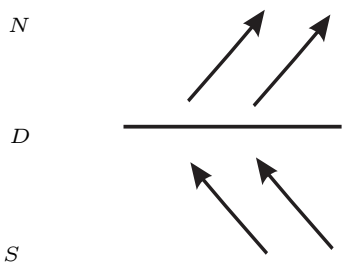

(a)

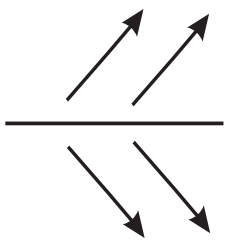

(b)

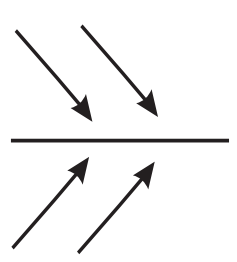

(c)

Figure 1: Arcs on $D$

Other developments in this direction can be found in Garcia-Sotomayor [5], where piecewise linear vector fields are studied and Buzzi-da Silva-Teixeira [3], where the method of singular perturbations are used to study certain discontinuous vector fields.

In this paper we deal with discontinuous vector fields $Z$ defined by a pair $(X, Y)$, where $X$ and $Y$ are polynomial vector fields in the plane. The goal here is establish conditions on $Z$ que determine when a singular point of $Z$ in $D$ is a center or a focus.

\section{Statement of the main results}

Let $f: \mathbb{R}^{2} \rightarrow \mathbb{R}$ be the function $f(x, y)=y$. We use $N, S$ and $D$ to denote the respective subsets of the plane $\{(x, y): y>0\},\{(x, y): y<0\}$ and $\{(x, y): y=0\}$. Therefore, we have that $D=f^{-1}(0), N=f^{-1}(0, \infty)$ and $S=f^{-1}(-\infty, 0)$.

The discontinuous vector fields $Z$ is defined by:

$$
Z(q)= \begin{cases}X(q) & \text { if } \quad f(q) \geq 0 \\ Y(q) & \text { if } \quad f(q) \leq 0\end{cases}
$$

where $X, Y$ are planar vector fields. We write $Z=(X, Y)$, where we will allow to be bi-valued at the points of $D$. Following Filippov's terminology (in [4]), we distinguish the following arcs in $D$ :

- Sewing $\operatorname{Arc}(S W)$ : characterized by $X f \cdot Y f>0$ (see Figure 1 (a)).

- Escaping Arc $(E S)$ : given by the inequalities $X f>0$ and $Y f<0$ (see Figure 1 (b)).

- Sliding $\operatorname{Arc}(S L)$ : given by the inequalities $X f<0$ and $Y f>0$ (see Figure 1 (c)).

As usual, here and in what follows, $X f$ will denote the derivative of the function $f$ in the direction of the vector $X$. i.e. $X f=\langle\nabla f, X\rangle$.

On the $\operatorname{arcs} E S$ and $S L$ we define the Filippov vector field $F_{Z}$ associated to $Z=(X, Y)$, as follows: if $p \in S L$ or $E S$, then $F_{Z}(p)$ denotes the vector tangent to $D$ in the cone spanned by $X(p)$ and $Y(p)$.

A point $p \in D$ is called a $D$-regular point of $Z$ if one of the following conditions hold:

i) $X f(p) \cdot Y f(p)>0$. This means that $p \in S W$;

ii) $X f(p) \cdot Y f(p)<0$ but $\operatorname{det}[X, Y](p) \neq 0$. This means that $p$ belongs either to $S L$ or $E S$ and it is not a singular point of $F_{Z}$.

Now, a point $p \in D$ is called a D-singular point of $Z$ if either $X f(p) \cdot Y f(p)=0$; or $X f(p) \cdot Y f(p)<0$ and $\operatorname{det}[X, Y](p)=0$.

We will call $p \notin D$ a real (respectively virtual) singular point of $Z=(X, Y)$ if either it is a singular point of $X$ and $p \in N$ or it is a singular point of $Y$ and $p \in S$ (respectively if either it is a singular point of $X$ and $p \in S$ or it is a singular point of $Y$ and $p \in N$ ). 
A continuous closed curve $\gamma$ consisting of two regular trajectories, one of $X$ and the other of $Y$, and two points $\left\{p_{1}, p_{2}\right\}=D \cap \gamma$ is called a $D$-closed orbit of type 1 (or simply $D$-closed orbit) of $Z$, if $\left\{p_{1}, p_{2}\right\}$ are sewing points and $\gamma$ meets $D$ transversally in $\left\{p_{1}, p_{2}\right\}$.

Let $p$ be a isolated $D$-singular point of the discontinuous vector field $Z$. If there exist a regular trajectory $\gamma$ of $X$ (respectively of $Y$ ) and a open set $V \subset N$ (respectively a open set $V \subset S$ ) such that $\gamma \rightarrow p$, either to positive or negative time, in $V$ we called $\gamma$ a $D$-characteristic orbit of $Z$ at $p$. We also saw que $\gamma$ is a D-characteristic orbit of $Z$ at $p$ if $p \in \gamma$ and either $\gamma \subset E S$ or $\gamma \subset S L$.

If $p$ is a isolated $D$-singular point of $Z$ and $Z$ has not $D$-characteristic orbit at $p$ we call it $D$-monodromic. A $D$-singular point $p$ of $Z$ is a center if there exist a neighborhood $V$ of $p$ such that $V$ is fulfilled of $D$-closed orbits of $Z$ with the unique exception of $p$. A $D$-singular point $p$ of $Z$ is a focus if $p$ is $D$-monodromic but it is not a center.

A $D$-singular point $p$ is a fold point of order $n$ of $X$ (respectively $Y$ ) if $n \in \mathbb{N}$ is even, $X f(p)=\cdots=X^{n-1} f(p)=0$ and $X^{n} f(p) \neq 0$ (respectively $Y f(p)=\cdots=Y^{n-1} f(p)=0$ and $Y^{n} f(p) \neq 0$ ), where $X^{n} f(p)=X\left(X^{n-1} f\right)(p)$. In this case we saw that $X$ (respectively $Y$ ) has a contact of order $n$ with $D$ at $p$.

Consider the planar differential system associated to discontinuous vector field $Z$ of the form

$$
\dot{x}=P(x, y), \quad \dot{y}=Q(x, y),
$$

where

$$
(P(x, y), Q(x, y))= \begin{cases}\left(P^{+}(x, y), Q^{+}(x, y)\right) & \text { if } y>0 \\ \left(P^{-}(x, y), Q^{-}(x, y)\right) & \text { if } y<0\end{cases}
$$

and so that $X=\left(P^{+}, Q^{+}\right)$and $Y=\left(P^{-}, Q^{-}\right)$. In what follows $P^{ \pm}$and $Q^{ \pm}$are linear functions on $\mathbb{R}^{2}$. This define the following linear differential systems

$$
\left(\begin{array}{c}
\dot{x} \\
\dot{y}
\end{array}\right)=\left(\begin{array}{c}
P^{+}(x, y) \\
Q^{+}(x, y)
\end{array}\right)=\left(\begin{array}{cc}
a^{+} & b^{+} \\
c^{+} & d^{+}
\end{array}\right)\left(\begin{array}{l}
x \\
y
\end{array}\right)+\left(\begin{array}{c}
\alpha^{+} \\
\beta^{+}
\end{array}\right)
$$

and

$$
\left(\begin{array}{c}
\dot{x} \\
\dot{y}
\end{array}\right)=\left(\begin{array}{c}
P^{-}(x, y) \\
Q^{-}(x, y)
\end{array}\right)=\left(\begin{array}{ll}
a^{-} & b^{-} \\
c^{-} & d^{-}
\end{array}\right)\left(\begin{array}{l}
x \\
y
\end{array}\right)+\left(\begin{array}{c}
\alpha^{-} \\
\beta^{-}
\end{array}\right) .
$$

We call (2) and (3) the northern and southern subsystem of (1) respectively. From [4] we know that the flow of $(1)$ denoted by $\varphi(t,(x, y))$ can be defined by using the flows $\varphi^{ \pm}(t,(x, y))$ of (2) and (3). For example, for a point $(x, y) \in N \cup S$, we have

$$
\varphi(t,(x, y))=\varphi^{+}(t,(x, y)) \text { if } \varphi^{+}(t,(x, y)) \in N,
$$

or

$$
\varphi(t,(x, y))=\varphi^{-}(t,(x, y)) \text { if } \varphi^{-}(t,(x, y)) \in S .
$$

For a point $\left(x_{0}, y_{0}\right) \in D$ satisfying $\left.X f \cdot Y f \quad\right|_{\left(x_{0}, y_{0}\right)}>\quad 0$, i.e. $Q^{+}\left(x_{0}, y_{0}\right) Q^{-}\left(x_{0}, y_{0}\right)>0$ we define $\varphi\left(t,\left(x_{0}, y_{0}\right)\right)$ as follows

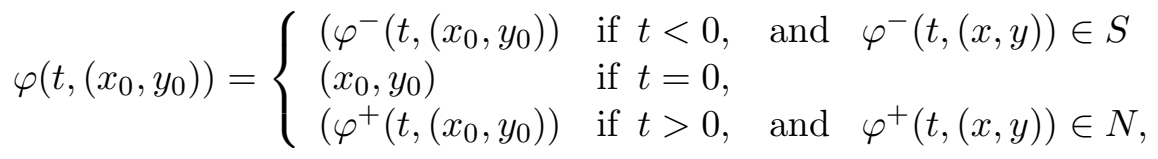

if $Q^{+}\left(x_{0}, y_{0}\right)>0$ and $Q^{-}\left(x_{0}, y_{0}\right)>0$; and

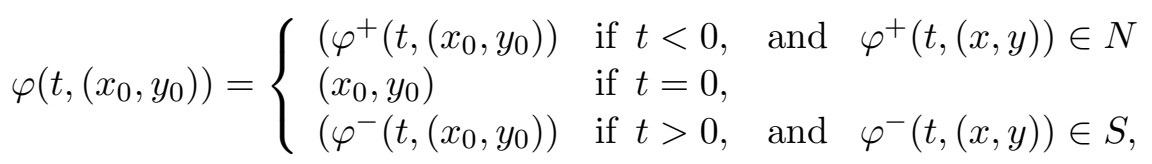

if $Q^{+}\left(x_{0}, y_{0}\right)<0$ and $Q^{-}\left(x_{0}, y_{0}\right)<0$. 
Since the study of linear differential systems is completely known from the works of Laplace in 1812, the only field of research in piecewise linear differential systems is about the orbits which move on both sides of line $y=0$. In this paper we are interesting in the possibility to have periodic orbits surrounding a $D$-singular point of $Z$, in particularly on the Center-Focus problem. As in the continuous case, this problem is also a challenge. In order if $Z$ has a $D$-closed orbit we can define a Poincaré map from $y=0$ to itself defined in $x \leq 0$, and another defined in $x \geq 0$. Every one of this Poincaré maps is of the form $\psi: I \rightarrow J$ with the (bounded or unbounded) intervals $I$ and $J$ of $y=0$ so that $\psi(I)=J$, then for $q \in I$ we have that $\varphi(t, q) \in J$ where the time $t$ may be different for each point of the interval $I$. The set of orbits going from the points of $I$ to the point of $J$ define what is called a transition flow. Thus, to have a closed orbits surround a $D$-singular point we must then have a transition flow $\psi^{+}: I^{+} \rightarrow J^{+}$and $\psi^{-}: I^{-} \rightarrow J^{-}$for each subsystem, so that the intersection of the respective segments $J^{+} \cap I^{-}$ and $J^{-} \cap I^{+}$are not empty. In this way we can define a Poincaré return map and determine the existence or not of closed orbits. In other words, a $D$-singular point is a center if and only if its Poincaré return map is the identity. Using the same idea we can also study the existence or not of limit cycles.

\section{Monodromic $D$-singular points}

In this section we give necessary and sufficient conditions to a $D$-singular point be a monodromic.

Proposition 1. Let $X$ and $Y$ be the respective vector fields associated to systems (2) and (3). If $p$ is a isolated D-singular point of $Z$ and $X f(p)=X^{2} f(p)=0$, then $p$ is a singular point of $X$. Analogously, if $p$ is a isolated D-singular point of $Z$ and $Y f(p)=Y^{2} f(p)=0$, then $p$ is a singular point of $Y$.

Proof. We have that $p=\left(x_{0}, 0\right)$, so that $X f(p)=c^{+} x_{0}+\beta^{+}$. Since $p$ is a isolated $D$-singular point of $Z$ and $X f(p)=0$, it follows that $c^{+} \neq 0$ and so $x_{0}=-\beta^{+} / c^{+}$, i.e. $p=\left(-\beta^{+} / c^{+}, 0\right)$. Now as

$$
X f(p)=Q^{+}(p) \text { and } X^{2} f(p)=c^{+} P^{+}(p)+d^{+} Q^{+}(p),
$$

we have that $X f(p)=X^{2} f(p)=0$ implies that $p$ is a singular point of $X$. In the same way we prove that if $p$ is a isolated $D$-singular point of $Z$ and $Y f(p)=Y^{2} f(p)=0$, then $p$ is a singular point of $Y$.

The following corollary is immediate of the above preposition.

Corollary 2. Let $X$ and $Y$ be the respective vector fields associated to systems (2) and (3). If $p$ is a fold point of $X$ (respectively $Y$ ), then $X$ (respectively $Y$ ) has contact of order 2 (i.e. parabolic contact) with $D$.

Proposition 3. Let $p$ be a $D$-singular point of $Z=(X, Y)$. If $p$ is a $D$-monodromic singular point, then $X f(p)=Y f(p)=0$ and there is a neighborhood $V$ of $p$ in $D$ with $\left.X f \cdot Y f\right|_{V}>0$ with the unique exception of $p$.

Proof. In fact, because if the necessary condition of proposition is not true, then $p$ has a $D$ characteristic orbit and so it is not a $D$-monodromic singular point of $Z$.

The following theorem classifies the $D$-monodromic singular points of $Z$.

Theorem 4. Let $Z=(X, Y)$ be a discontinuous vector fields. We suppose that $p$ is a D-singular point of $Z$ such that $X f(p)=Y f(p)=0$ and $\left.X f \cdot Y f\right|_{D}>0$ in a neighborhood of $p$ in $D$ with the unique exception of $p$. 
i) If $X(p) \neq 0$ and $Y(p) \neq 0$, then $p$ is a D-monodromic singular point of $Z$ if and only if it is a fold of order $n$ of $X$ with $X^{n} f(p)<0$ and it is a fold of order $m$ of $Y$ with $Y^{m} f(p)>0$.

ii) If $X(p)=0$ (respectively $Y(p)=0$ ) and $Y(p) \neq 0$ (respectively $X(p) \neq 0)$, then $p$ is a $D$ monodromic singular point of $Z$ if and only if $p$ is a monodromic point of $X$ (respectively $Y$ ) and $p$ is a fold of order $n$ of $Y$ (respectively $X$ ) with $Y^{n} f(p)>0$ (respectively with $\left.X^{n} f(p)<0\right)$.

iii) If $X(p)=Y(p)=0$, then $p$ is a D-monodromic singular point of $Z$ if and only if $p$ is a monodromic point of $X$ and $Y$.

Proof. As $p$ is a $D$-singular point of $Z$ such that $X f(p)=Y f(p)=0$ and $\left.X f \cdot Y f\right|_{D}>0$ in a neighborhood of $p$ in $D$ with the unique exception of $p$, it follows that $p$ is a isolated singular point of $Z$. Now, $p$ is a $D$-monodromic singular point of $Z$ if and only if it is isolated and $Z$ has not $D$-characteristic orbit at $p$. Therefore, the hypothesis and conditions stated in each case ((i), (ii) and (iii)) are necessary and sufficient to nonexistence of $D$-characteristic orbits of $Z$ at $p$. This finish the proof of theorem.

\section{References}

[1] A. Andronov, V. Chaikin et al., Theory of Oscillators, Addison, Addison Wesley (1966).

[2] A. Andronov, I. Gordon, E. Leontovich and G. Maier, Theory of Bifurcations of Dynamical Systems on a Plane, Israel Program for Scientific Translations, John Wiley, New York (1973).

[3] C. Buzzi, P. da Silva and M. Teixeira, A singular approach to discontinuous vector fields on the plane, J. Differential Equations 231, 2 (2006), 633-655.

[4] A.F. Filippov, Differential Equations with Discontinuous Righthand Sides, Kluwer Academic, Netherlands, 1988.

[5] R. Garcia and J. Sotomayor, Structural stability of piecewise-linear vector fields, J. Differential Equations 192, 2, (2003), 553-565.

[6] E. González Velasco, Generic Properties of Polynomial Vector Fields at Infinity, Trans. Amer. Math. Soc. 143 (1969), 201-222.

[7] V.S. Kozlova, Roughness of a Discontinuous System, Vestnik Moskovskogo Universiteta, Matematika 5 (1984), 16-20.

[8] A.L.F. Machado and J. Sotomayor, Structurally Stable Discontinuous Vector Fields in the Plane, Qual. Th. Dynamical Systems 3 (2002), 227-250.

[9] M.M. Peixoto, Structural Stability on Two-dimensional Manifolds, Topology 1 (1962).

[10] J. Sotomayor, Stable Planar Polynomial Vector Fields, Rev. Mat. Iberoamericana 1, 2 (1985), 15-23.

[11] J. Sotomayor, Curvas Definidas por Equações Diferenciais no Plano, Colóquio Brasileiro de Matemática, IMPA, Rio de Janeiro (1981).

[12] J. Sotomayor and M.A. Teixeira, Regularization of Discontinuous Vector Fields, International Conference on Differential Equation, Lisboa (1995), 207-223. 